\title{
El retiro digno del médico
}

Enrique Wolpert-Barraza, ${ }^{1 *}$ Alejandro Alfonso-Díaz, ${ }^{2}$ Juan Felipe Sánchez-Marle, ${ }^{3}$

Laura Elena Jáuregui-Camargo 4 y Francisco Moreno ${ }^{4}$

${ }^{1}$ Dirección del Cuerpo Médico; ${ }^{2}$ Dirección General; ${ }^{3}$ División de Cirugía; ${ }^{4}$ Medicina Interna. Centro Médico ABC, Ciudad de México, México

\section{Resumen}

El retiro del médico de su actividad profesional es un tema de interés global. En México no existe una regulación oficialque determine la edad a la que el médico debe retirarse de su profesión como sucede en otros países. En este simposio se presentan las medidas realizadas en el Centro Médico $A B C$ en los últimos años en lo que al retiro médico se refiere, cồn el establecimiento de un comité. Lo anterior se ha logrado en parte también con la creación de prácticas médicas grupalešque integran a los médicos en sus distintas especialidades, cuya intención, entre otros aspectos de interés individual y colectivo, es el establecimiento de un fondo para el retiro.

PALABRAS CLAVE: Autoestima. Dignidad. Jubilación.

\section{Abstract}

Retirement of physicians from their professional activity is a subject of global interest. In Mexico, there is no official regulation that determines the age at which physicians should retire from their profession as in other countries. This symposium presents the measures implemented at the ABC Medical Center in recent years about physicians' retirement, with the establishment of a committee. This has been achieved in part also with the creation of group medical practices that integrate doctors in their different specialties, whose interest among other aspects of individual and collective interest, is the establishment of a retirement fund.

KEY WORDS: Self-esteem. Dignity. Retirement.

Correspondencia:

*Enrique Wolpert-Barraza

E-mail: ewolpertb @abchospital.com
Fecha de recepción: 18-09-2019

Fecha de aceptación: 30-09-2019

DOI: 10.24875/GMM.19005545
Gac Med Mex. 2019;155:647-655 Disponible en Pǘmed www.gacetamedicademexico.com 


\section{Introducción}

El retiro del médico de su actividad profesional es un tema de interés global. En México no existe una regulación oficial que determine la edad a la que el médico debe retirarse de su profesión, como sucede en otros países. El Instituto Mexicano del Seguro Social, por ejemplo, en su Contrato Colectivo de Trabajo señala:

Los trabajadores con 30 años de servicio en el Instituto, sin límite de edad, que deseen jubilarse, podrán hacerlo con la cuantía máxima que otorga el Régimen. A las trabajadoras con 27 años de servicio se les computarán tres años más para efectos de jubilación.

En el Ejército Mexicano, el retiro del médico no es solo en función de la edad sino también del grado militar que haya alcanzado, pero desde luego, una vez jubilado del ejército puede seguir ejerciendo en forma privada.

En los siguientes trabajos presentaremos las acciones realizadas en el Centro Médico $A B C$ en los últimos años con el establecimiento de un comité para el retiro médico. Nos hemos preocupado porque el médico, desde que inicia su práctica profesional en la institución, tenga en cuenta la posibilidad de dedicar parte de sus ingresos para que cuando llegue el retiro, este sea con la dignidad con la que ha vivido. Lo anterior se ha logrado en parte también con la creación de prácticas médicas grupales que integran a los médicos en las distintas especialidades que cultivan y donde, entre otros aspectos de interés individual y colectivo, está el establecimiento de un fondo para el retiro.

En México hay varios artículos sobre el tema, entre los que destacan las publicaciones de Rafael Muñoz Kapellman, con reflexiones como la siguiente:

Que la cirugía es ciencia y arte, que demanda que el cirujano reúna características muy especiales y bien definidas, como vocación, temperamento, inteligencia, valor, audacia, criterio, decisión, destreza manual, fortaleza física, amor al estudio, así como bases morales y éticas muy sólidas.

Muñoz también menciona:

[...] que en términos generales es razonable suponer que la plenitud de la mayoría de los cirujanos dure hasta los 65 años y posteriormente, y dependiendo el momento de cada caso particular, se iniciará inexorablemente la disminución progresiva y persistente de las facultades mentales y físicas, proceso imposible de evitar.

En el primer número de 2018 de Anales Médicos de la Asociación Médica del Centro Médico ABC, Octavio Ruiz Speare publicó un artículo de por qué se debe retirar el cirujano, cuándo y en qué condiciones, en el que plantea las siguientes preguntas: ¿cómo afecta el juicio y la habilidad del cirujano con el envejecimiento?, ¿el cambio es igual en todos los cirujanos? Es importante la evaluación que hace un cirujanê de sí mismo, la autoevaluación y conocer objetivamente el juicio y las habilidades del cirujano y, desde luego, las opciones para realizarlas una vez que se retira.

En este simposio presentamos varios temas ê que al retiro médico se refiere: identificación dê un problema real en el Centro Médico ABC, Comitếdel Retiro Médico del Centro Médico ABC, educación para el retiro del médico y avances en la planeación del retiro digno del médico en el Centro Médico ABC.

\section{Identificación de un problema real en e Centro Médico ABC}

El problema del retiro digno no es exclusivo délos médicos (doctoras y doctores) ni de México, eš un problema que encontramos en otros profesionales $y$, desde luego, como un asunto común de la población en general es un síntoma del subdesarrollo, por lo que también lo padecen otros países.

Tratar de reducir el problema del retiro digno ya sea en medicina o en cualquier otra profesión, tema económico es un error pues es un tema que tiene más aristas. Aceptando lo anterior, es importante reconocer que los otros factores que involucran la decisión del retiro no podrán llevarse a cabo adecuadamente sin un plan de jubilación económico conveniente y oportuno.

Pienso que el problema inicia con una falta de eultura y aceptación que consiste en reconocer que llegará el día en que tendremos que cerrar un capitulo profesional en nuestras vidas y que este hecho iñevitable, desde el aspecto profesional y personal, tanto para el individuo como para aquellos que lo rodean, es trascendental.

En nuestra juventud temprana no se nos ha inculcado que nuestra vida productiva llegará a un fin̄al y que, por lo tanto, debemos tener en cuenta que hăbrá el momento en que enfrentaremos la opción de rêtito y la necesidad de trabajar con el fin de estar preparados para ese momento.

Es evidente que el problema es generacional en el sentido de que se hace evidente en una generación específica, y no porque las anteriores sí hayan entendido el significado de un retiro digno y hayan trabajado para ello, sino que se presenta en la generación 


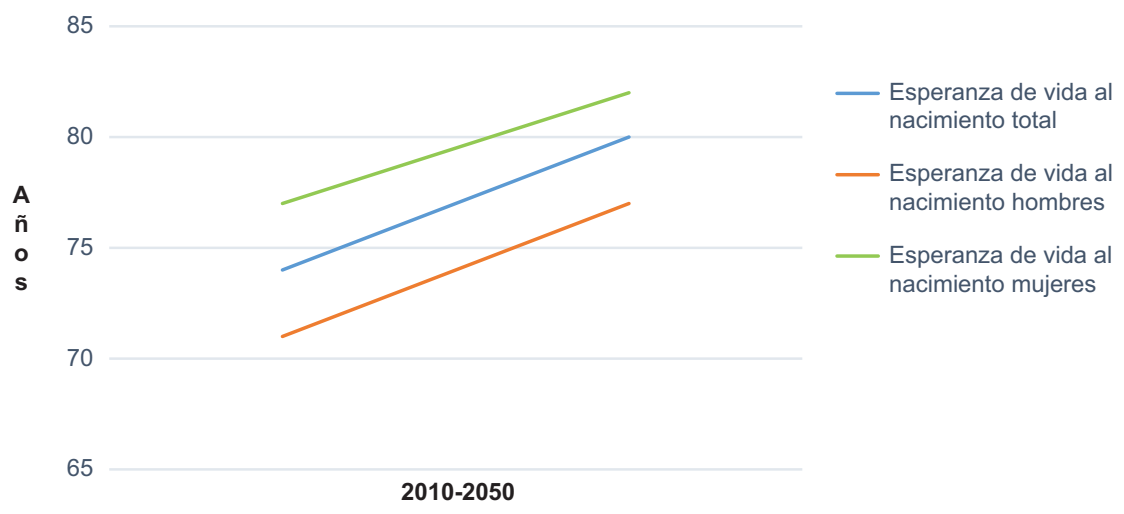

Figura 1. Expectativa de vida en México 2010-2050.

que empezó a vivir más allá de los años que inicialmente se establecieron como "la edad de retiro" y que se determinó en la primera mitad del siglo pasado en los 65 años de edad; en 1950 la expectativa de vida en México era de 47 años, 10 años más tarde, en 1960, apenas superaba los 60 años, por ello, las generaciones de esos años fueron los padres de muchos que hoy enfrentan la decisión del retiro, quienes nunca tuvieron que ahorrar para el retiro porque en sus hogares nunca se vivió con personas retiradas ni se vio qué tipo de actividades podía llevar a cabo alguien en esa etapa de la vida. Simplemente nuestros padres y abuelos, salvo algunas excepciones, enfermaron y murieron antes de llegar a la edad de retiro.

Hoy, la esperanza de vida promedio supera los 75 años y para el 2050 llegará a los 80 , siendo para las mujeres mayor que para los hombres.

En la población que ha tenido acceso a servicios de educación y salud adecuados, la probabilidad de vivir hasta los 90 años es de $50 \%$ para quienes han nacido en este siglo y para quienes tienen acceso a condiciones altas de educación y salud esta esperanza aumenta a 105 años (50\%); hace 100 años esta probabilidad era de $1 \%$ (Figura 1).

Lo anterior hace evidente lo siguiente:

- La edad de retiro no puede seguir siendo a los 65 años.

- El alargamiento de la edad de retiro tiene consecuencias económicas relevantes, como incremento en las tasas de desempleo para las generaciones más jóvenes, afectaciones en las tasas de interés, ya que las personas jubiladas no ahorran, etcétera.

- Hay que replantear a nivel individual y colectivo los esquemas de retiro existentes, ya que los actuales son insuficientes, entre otras razones por el alto porcentaje de personas que participan en la economía informal y no aportan dinero para su jubilación y atención sanitaria en los sistemas gubernamentales.

\section{El plan de jubilación en el Centro Médico ABC}

Con los años, el problema se hizo evidente por el conocimiento de algunos médicos que a cierta edad se encontraban sin recursos económicos para hăcer frente a la caída en sus ingresos por la disminución del flujo de sus pacientes y la falta de un plam de protección integral para afrontar las necesidaddes económicas y de atención de salud de ellos y dē su cónyuge.

Una de las acciones que se tomaron en los primeros años de este siglo fue la creación del Plan "Doêtor Bernardo Tanur" (llamado de esta forma en honor al doctor Bernardo Tanur Tatz, presidente del cuerpo médico del ABC de 2001 a 2007, quien promovió la idea) que consiste en garantizar con fondos del propio Ceñtro de Médico y de la Asociación Médica que los médicos que cumplen ciertos requisitos de edad y años de práctica médica en el ABC tengan acceso a servicios médicos y hospitalarios. Si bien es un plan novedoš y atractivo, tiene ciertos límites y está lejos de soluciönar el problema de un retiro digno, ya que se enfoca en la atención de los médicos en caso de enfermedad.

En 2006, el ABC decidió crear un esquema cônocido como Práctica Médica Grupal y para ello llevó a cabo un análisis de la relación entre los médicoscon la administración del Centro Médico y la problemática de la práctica médica en un centro médico privado.

Como resultado de este análisis se confirmó que, entre otros aspectos por resolver, había que promôver que los integrantes de los distintos conjuntos de 
Práctica Médica Grupal participaran en la creación de fondos de pensiones como una forma de financiar las prestaciones y retiro de los médicos participantes.

A pesar de lo evidente de la falta de planes de previsión para el retiro, la idea de crear fondos para el momento de jubilarse no fue recibida como positiva por una parte importante de los miembros de la Práctica Médica Grupal de Neurociencias, la primera en formarse. Parte de esta falta de aceptación consistía, por un lado, en la percepción de que el Centro Médico $A B C$ pretendía influir en el uso y destino del dinero ganado por los médicos y, por otro, en la falta de información sobre el funcionamiento de estos fondos, además de la creencia equivocada de que estos deben ser de un importe tal que permita su deducción para efectos del pago del impuesto sobre la renta. Con el tiempo, ambas preocupaciones se han ido superando.

Un punto relevante y que el doctor Francisco Moreno ha tocado en diversas ocasiones con sus colegas y en distintos foros, es que el monto económico que se dedica a formar el fondo de pensiones no obtendrá rendimientos financieros muy altos; la razón es senciIla, cualquier inversión que aspira a obtener altos rendimientos estará sujeta a alto riesgo, regla universal en materia financiera: a mayor rendimiento, mayor riesgo. El fondo de pensiones debe obtener un rendimiento que garantice el valor adquisitivo a través del tiempo pero que no ponga en riesgo el capital que se vaya acumulando; es un capital que se invierte a largo plazo para los años en que no obtengamos un ingreso importante por la actividad profesional que realicemos y, por lo tanto, a poco riesgo, habrá poco rendimiento.

Lo anterior no significa que seamos ineptos en el manejo de nuestros fondos, sino que el monto que dediquemos a ahorrar para nuestro retiro debe manejarse con un criterio prudencial e inteligente, de tal manera que nos proteja al momento de jubilarnos; el monto que se dedica estará sujeto a diversos factores como el ingreso que se percibe durante nuestra vida productiva, el número de años que estimamos ser productivos y la esperanza de vida, entre otros, pero nunca en función de los montos que las leyes en materia de impuestos que la Secretaría de Hacienda y Crédito Público nos permita hacer deducibles.

\section{Comité del retiro médico en el Centro Médico ABC}

El aumento en la longevidad de los seres humanos ha hecho necesarias ciertas evaluaciones del desempeño de los individuos para determinar si continúan siendo aptos en el desarrollo de sus actividades profesionalles. En medicina esta valoración debe ser muy cuidadosa.

En el Centro Médico ABC, durante el año 2010 se llevó a cabo un taller tipo FODA para el desarrolle de una planeación para el retiro del médico.

Como estrategia para resolver esta debilidađi se determinó la formación de un Comité para la Planeación del Retiro, presidido por el doctor Juan Fềipe Sánchez Marle y con la participación de otros siete médicos, que cubrían edades de la cuarta a la octava década de la vida, así como distintas especialidades medicoquirúrgicas y con interés en el tema.

El Comité se reunió mensualmente para investigar primero antecedentes relacionados con el problema. En la literatura nacional se encontró un artículo eșcrito en 1998 por el doctor Muñoz Kapellmann y un simposio en la Academia Nacional de Medicina coordinado por el doctor Fajardo Dolci en septiembre de 2011, titulado "El Retiro Médico". Se estableció que en las instituciones de salud no había una edad precisa para retirarse obligatoriamente, excepto ę el Servicio de Sanidad Militar. En la medicina privadą no existe ninguna regulación, solamente la conciencia del médico y los resultados de su actuación profésional, cuando el hospital donde trabaja los registre adecuadamente.

En Estados Unidos el tema es de gran actualiēad. El Colegio Americano de Cirujanos ha establecido que una edad precisa para el retiro del médico no puede ser puntualizada, por las variables de aptitudes de los individuos aun de la misma edad. Se encontró que evaluaciones periódicas de los médicos sería el mejor método para determinar sus aptitudes presentes. Este método demostró que a mayor edad, menor posibilidad de conservar las facultades en estadoóóptimo, lo que aconsejó que la frecuencia de estas èvaluaciones aumentara conforme la edad fuera mayor.

Con el antecedente de no poder establecer una edad para el retiro y la necesidad de efectuar evaluaciones periódicas al cuerpo del médico, se plantearon para el Comité los siguientes objetivos:

1. Proponer planes para el retiro entre los médiços de nuevo ingreso al cuerpo médico $A B C$.

2. Difundir la cultura del retiro entre los médicos del cuerpo médico ABC.

3. Establecer las normas a las que se sujetarán las evaluaciones periódicas para fijar los privilegios medicoquirúrgicos de los que puedan haceruso los médicos en las instalaciones del Centro MMédico ABC. 
4. Informar al Comité de Competencia Médica de los resultados de las evaluaciones.

La decisión de retirarse de la medicina es personal, está basada en la autocrítica de la propia práctica profesional, pero influida por el deseo de seguir ejerciendo la profesión, por no haber planificado el retiro y porque la práctica médica es la única o la principal fuente de ingresos.

A los médicos jóvenes se les inicia en la cultura del retiro. Deben pensar y planear en esa etapa de la vida. Al mencionar el tema, muchas veces los médicos mayores se molestan porque se dan cuenta de que el tiempo llega y no se prepararon para ello. Las limitaciones emocionales y económicas en algunos pueden entorpecer sus decisiones sobre su ejercicio médico, poniendo en peligro su prestigio y aumentando las, posibilidades de problemas legales y riesgos para sus pacientes y la institución en la que laboran. Solo evaluaciones periódicas por el hospital donde trabajan normarán su capacidad de ejercer satisfactoriamente su profesión.

Los hospitales donde los médicos trabajan tienen la capacidad de evaluarlos en tres esferas. Hemos dejado la tarea en manos de los consejos de especialidades médicas. Los médicos deben estar certificados y recertificados puntualmente. Se ha propuesto evaluar la esfera psicomotriz en el laboratorio de simuladores y se adaptará a la especialidad médica examinada. Por último, la esfera afectiva se evalúa por el resultado de relaciones interpersonales, quejas, complicaciones, demandas y, más recientemente, volúmenes de trabajo.

\section{Educación para el retiro del médico}

En mi opinión, el médico, ingeniero, arquitecto, piloto y abogado debemos tomar con mucha seriedad la decisión del momento oportuno para retirarnos, ya que a diferencia de otras profesiones (artistas, deportistas, chefs) nos comprometemos a proteger los valores más preciados del ser humano: la salud, la vida y la libertad.

Para el médico, el retiro del ejercicio profesional es tan certero como la muerte para el que nace. El retiro y la muerte son temas frecuentemente evadidos por considerarlos dolorosos y lejanos. Sin embargo, el conocimiento profundo de la etapa del retiro permite cambiar esa sombría perspectiva y convertirla en una meta incluso deseable. Es la educación en el retiro lo que nos permitirá planear esa etapa con el mismo entusiasmo con el que planeamos un gran viaje, un reto deportivo o la organización de nuestro primer consultorio o clínica.
Debo reconocer que yo pensé por primera vez en mi retiro al salir del cine, después de haber visto la película The Bucket List (en México Ahora o nuñca), con Jack Nickolson y Morgan Freeman; ambos personajes de la tercera edad se encuentran en un "rêtiro forzoso" por padecer una enfermedad terminal yeentonces deciden hacer lo que siempre quisieron $\Phi$ no habían podido lograr.

Yo me acercaba a los 40 años y había logrădo completar mi primera bucket list: una muy satisfaetoria carrera académica, una actividad profesionâf en crecimiento, una hermosa familia y un patrimonio en formación; sin embargo, entré en pánico al darrme cuenta de que lo que seguiría en mi vida sería muy aburrido si tan solo me concentraba en mantener lo hasta entonces logrado y fue peor aún el sentimiênto al darme cuenta de que poco de todo eso me acómpañaría hasta el final de mi vida. Lo aprendido entonces dejaría de ser vigente, mi actividad profesional sería rebasada por colegas más jóvenes y⿳亠mmis hijas crecerían; fue entonces cuando decidí hācer una nueva lista y empezar los preparativos para alcanzarla.

\section{Crear conciencia}

Hace algunos años, el doctor Sánchez Marle đame invitó a formar parte del recién creado Comité para la Planeación del Retiro Médico en el Centro Méddico $A B C$, desde donde nos dirigió para estudiar el tema dentro de nuestra institución y nos permítió, tomando en cuenta lo que se realiza en otras instituciones y profesiones, hacer un planteamientoresolutivo para implantar poco a poco entre nuestros colegas.

Dicho planteamiento incluyó una primera encuěsta en la que se estudió el impacto que tenía entre nūestros pares el tema, el nivel de conocimiento de este y la planeación que tenían para enfrentarlo. Con esos resultados nos propusimos como primer paso difundir el tema, publicamos artículos, se hicieron preseñtaciones en diferentes foros dentro del hospital como la sesión general, la sesión de la asociación médiça y en el Congreso Anual de la Sociedad Médica. Tămbién se instituyó dar una plática durante el curso de inducción que se imparte a todos los médicos de nuevo ingreso en nuestra institución.

En este primer paso, el principal objetivo era "hablar del indeseable tema", hacer conciencia de su irremediable existencia, de la necesidad de aceptar la lidad y de preparase para ella. 


\section{Plantear escenarios}

En una segunda etapa, analizamos los diferentes escenarios en los que un médico se "retira". Hay quienes se retiran al morir o los que una enfermedad los retira; sabemos de quienes un error médico les costó la licencia o quienes van "perdiendo la fama". La intención de reconocer estos tristes escenarios es evitarlos; ningún colega merece ese final después de haber sacrificado tanto tiempo de sueño, familia y amigos durante años.

Esto nos llevó a visualizar los posibles escenarios para un retiro digno del médico y así planear la preparación que se requiere para lograrlo. El escenario ideal es, sin duda, el voluntario, aquel al que llegamos por decisión propia, conservando la reputación y aprecio de pacientes y pares; en este podemos aprovechar el conocimiento, la experiencia y el tiempo para enseñar, para escribir, dar conferencias y asesorar a médicos en formación. Si la situación financiera lo amerita, la creación de grupos de trabajo, formados por médicos de la misma especialidad, permite que los médicos jóvenes aporten conocimientos actuales y los médicos más grandes enseñen la experiencia adquirida, mientras comparten el ingreso económico que generan entre todos. En este escenario también se favorece el retiro digno de los médicos de mayor edad.

En definitiva, el mejor escenario es aquel que nos permita estar en una situación física y emocional óptima que garantice disfrutar el entorno familiar y social sin angustiarnos por el sustento económico.

\section{Conocer "el retiro"}

Educar para el retiro nos obliga a entender cada parte que lo conforma. Con una mayor esperanza de vida seguramente nos tocará vivir varios años en el retiro. De manera que este sea placentero para el médico y su familia debemos procurar conservar cuatro aspectos fundamentales: salud física, salud emocional, salud financiera y salud social.

La educación en estos aspectos debería empezar en la facultad de medicina, sin embargo, durante toda esa etapa de formación (y peor aún durante los programas de residencias médicas) es cuando más atentamos en contra de nuestra salud física y emocional, descuidamos a la familia y amigos y carecemos de un ingreso suficiente que nos permita una situación económica estable.

El Centro Médico ABC fomenta la conservación de estos aspectos procurando la buena alimentación de sus médicos y enfermeras, revisando periódicamente los menús que se sirven en el restaurante, promüeve la realización de ejercicio en los gimnasios dentrô del campus, organiza eventos deportivos como carrerăs y caminatas de 5 y $10 \mathrm{~km}$, torneos de golf y sesiones culturales semanales para favorecer el equilibrio empcional y social. Finalmente, el doctor Francisco Moreno, actual presidente del Comité del Retiro, logró que todos los asociados interesados accedieran a un cursê de educación financiera y creó dentro de una prestigiada firma un fondo de ahorro para el retiro al que todosilos asociados tenemos acceso y buenos rendimientos?

\section{Cambiar percepciones}

Sin duda la parte más difícil de "educar" a los colegas en este tema ha sido cambiar la percepción que se tiene de que el retiro es una decisión personal y depende de cada uno.

Hace algunos años, en este mismo recinto, se reunieron grandes médicos como los doctores Juan hamón de la Fuente, José Kuthy Porter, Horacio Jiahich y Rafael Muñoz Kapellmann y presentaron diferentes aspectos de este tema. El doctor Kuthy presentóguna interesante reflexión acerca de los aspectos éticos relacionados con la decisión que debiera tomar cada médico, basándose en una profunda y honesta sión personal de las habilidades perdidas. El doettor Jinich compartió su proceso personal de entendimiento y aceptación del retiro y la forma como él mismo decidió llevarlo a cabo. El doctor Muñoz presentó diferentes escenarios en los que un médico ciruiano puede retirarse $y$, finalmente, el doctor De la Fuente explicó los aspectos emocionales que genera enfreentar esta situación, como tantas otras, en las que reconocemos una pérdida que genera miedo, tristěza, frustración y angustia.

Más recientemente, los doctores Enrique Wolpert y Octavio Ruiz Speare han escrito acerca de lo que se ha establecido en otras instituciones y en el extranjero y han utilizado este marco de referencia para diseñar un mecanismo menos subjetivo para tomar esta decisión, creando el concepto de una "evałuación completa de las capacidades médicas". La ămplia experiencia del doctor Wolpert en la evaluaçión para la certificación de los médicos, según sus conocimientos actuales relacionados con su especialidad, permitió extender este concepto a la "evaluacióri de las otras capacidades" que todo médico debe tener, conservar y actualizar para el buen ejercicio dê su especialidad. 
Así, con la creación del Centro de Simuladores y bajo la dirección del doctor Ruiz-Speare se logró complementar dicha evaluación, con la intención de ayudar a los médicos a medir sus habilidades y destrezas y poder planear con más objetividad su retiro, y permitir que la institución disponga de información objetiva acerca de la capacidad del médico evaluado de continuar ejerciendo. Esta evaluación integral está propuesta a partir de los 65 años y deberá repetirse periódicamente dependiendo de los resultados obtenidos en la evaluación previa, las incidencias ocurridas y la edad alcanzada.

\section{Cómo lograrlo}

De tal forma, es de vital importancia que todo médico en el ejercicio de su especialidad y miembro de alguna sociedad tenga acceso a estos recursos desde el inicio de su práctica, el acceso al conocimiento previo de que su actuar profesional deberá ser evaluado periódicamente con la sana intención de protegerle a él o ella y a la institución que le avala, y que estas herramientas le servirán en un futuro para saber que el momento de retirarse se acerca.

Deberá tener oportunidad, en su centro de trabajo, de procurarse una sana alimentación, un espacio y tiempo para el ejercicio físico, disponer de suficientes horas de descanso y relajación para compartir con la familia y amigos. Y, finalmente, contar con asesoría financiera desde el inicio del ejercicio de la profesión para entender la importancia del ahorro para el retiro.

Educar para el retiro médico implica difundir el tema entre los pares, dar a conocer los diferentes escenarios posibles, presentar los cuatro aspectos fundamentales (físico, emocional, financiero y social) que debemos procurar para disfrutar de esta etapa y, sobre todo, cambiar la percepción de que ésta sea una etapa negativa.

El doctor Nicolás Martín del Campo indica, acertadamente, cómo integrar la etapa del retiro en el plan de vida permitirá al adulto joven identificar los gustos y actividades como pintar, escribir, cocinar, tocar música o aprender otro idioma; qué disfrutará realizar en esta etapa y cómo la práctica de buenos hábitos de alimentación, la actividad física y el ahorro constante desde el principio de nuestra práctica médica nos permitirán lograr la autonomía necesaria durante la vejez.

Promover este conocimiento acerca de qué es el retiro y cómo prepararse integralmente para ese momento es lo que hará que dicho retiro sea digno y ligero. Cómo lograrlo:

- Enseñar la importancia del tema en escuelaș de medicina y residencias.

- Promover la salud física, mental, social y fiñanciera en los centros de trabajo.

- Hablar con la familia, amigos y pares del escenario en el que quisiera uno retirarse y conocer el método de evaluación adecuado para nuesttra práctica.

- Hacer, revisar y ajustar nuestra propia buckettlist permitirá que planeemos con gusto todo lo neccesario para disfrutar esta etapa.

Avances en la planeación del retiro digno del médico en el Centro Médico ABC

Cuando tomé el puesto de presidente del Comité del Retiro Médico, lo primero que noté es que no hablar del retiro es hablar de la falta de dinero yo es importante entenderlo. El retiro del médico incluye el aspecto económico, social, ocupacional y emocional; al investigar más sobre esto nos dimos cuenta de que el médico tiene que entender que vive para la medicina durante la mayor parte de su vida, pero algúñ día la medicina dejará de ser parte de ella y que tiene que aprender que antes de médico es ser humā̄o.

Respecto a la expectativa de vida, los principàles culpables de esta expectativa de vida mayor el trabajo somos nosotros mismos, el aumento de lassupervivencia nos ha hecho víctimas de nuestro propio éxito. Lo primero que platicamos con los alumnos médicos que se van a dedicar a ejercer su vida en la medicina es qué piensan hacer después de los 65 y cómo piensan hacerlo; qué han hecho hasta el día de hoy y qué han dejado de hacer, porque la mayoría de ellos no piensa en eso, sino que piensa en preseñnte.

Al médico le cuesta trabajo retirarse porque, comparado con otras profesiones, su carrera es muy larga, sobre todo ahora que hay especialidad, subespecialidad y megasubespecialidad; termina de estudiar la carrera alrededor de los 30 o 35 años. En ese momento sale a la calle y no tiene un letrero que diga $P h D$, por ello tiene que desarrollar sus habilidades para que sus pacientes empiecen a confiar en él; ësto le lleva otros cinco o 10 años, entonces, de pronto, aunque ya llegó a la cumbre profesional, ya tiene 45 años y ya solo le quedan 20 años tiempo eñ el que tiene que disfrutar y ahorrar para que los próximos 20 años después pueda vivir sin la necesidad de depender de la medicina. 
La ventana de oportunidad para lograr un sustento económico que nos permita vivir después de no ser médicos tenemos que forjarla desde que salimos de la carrera. Nuestra cumbre profesional debe ser el momento ideal en el que planeemos el plan B y, evidentemente, tener presente que si nuestros pacientes son primero, habrá un día en que no seremos el mejor médico que los pueda atender, en ese momento debemos tener la suficiente autocrítica para decir que hay alguien mejor. En resumen, en cuanto a la economía, el inicio de la vida productiva del médico es tardío, el periodo productivo es corto y la expectativa de vida aumenta, por lo tanto, ahorrar es la solución para vivir sin tener un trabajo a edad avanzada.

También hay cuestiones sociales, los médicos estamos dedicados totalmente a la medicina, el promedio de horas de trabajo es mucho mayor a las 48 horas semanales habituales y tenemos horarios muy irregulares; tenemos problemas para viajar, no tenemos facilidades para tomar vacaciones, tenemos una exigencia en cuanto a la actualización. En una encuesta que hizo la American College of Surgeons de 2000 médicos, solo $10 \%$ de los médicos tenía una afición, es decir, $90 \%$ va a llegar a esa edad y no va a saber qué hacer.

El profesional médico se suicida $28 \%$ más que cualquier otro, la tasa de suicidios aumenta, a diferencia de otras profesiones, cuando se llega a los 60 años. La depresión ocurre más o menos en $25 \%$ de los estudiantes de medicina, en $30 \%$ de los residentes y baja discretamente cuando se está en la cumbre profesional, pero lo que es increíble es que dos de cada tres médicos después de los 60 años están deprimidos; los médicos somos de las profesiones que más se divorcian.

¿Cuánto tenemos que ahorrar? Con lo que vivimos actualmente necesitamos empezar a ahorrar desde que salimos de la carrera de medicina. Es muy importante dar esta plática en las escuelas de medicina porque no nos percatamos de esto hasta que llegamos a los 55 o 60 años. A los 45 años deberíamos tener ahorrado dos veces todo el ingreso anual; a los 55 , cinco veces y a los 67, edad que marca American College of Surgeons como la edad en la que debería retirarse el médico, ocho veces el ingreso anual. En ese sentido, el retiro económico debe empezar ahora y, en cuanto al ámbito social, tener una afición, dedicar tiempo a los amigos debe empezarse en la cumbre profesional.

Desarrollamos este programa para el retiro del médico que se llama Forma Capital, instrumento que, a través de las prácticas médicas grupales, logra que todos los médicos ingresemos a un fondo único de manera que parte del dinero que nosotros recibimos automáticamente se va a ese fondo y al haceếun fondo único de todos los médicos logramos que ưna compañía importante nos dé un plan de rêtiro adecuado.

En 2016 hice una encuesta: a los médicos que entraban al hospital les daba una hoja, donde tenían đue poner su edad, sexo, si tenían un fondo de ahorio y a los cuántos años planeaban retirarse: 13 de las personas que contestaron eran mayores de 60 años, 14 estaban entre los 50 y 49 años y 13 más entrêlos 40 y 49 años. Esta encuesta se hizo en el Centro Médico ABC Campus Observatorio que, por cuestiones epidemiológicas, fue el primer hospital. Después fue el Campus Santa Fe, entonces había 38 hombres y dos mujeres, pero las respuestas fueron increíbles: 11 dijeron sí tener un fondo de retiro, pero 22 dijeron que creían tener un fondo de retiro, cuando sêles cuestionaba por qué creían tener un fondo de retiro, su respuesta era que tenían algún bien que podían vender para vivir de eso en el retiro; solo siete fueron muy honestos y dijeron que no tienen un fondo de retiro. Respecto a la edad a la que pensaban retirarse, 13 dijeron que a los 75 , siete dijeron que a los70, siete fueron muy claros al decir "cuando me muềy", siete dijeron que a los 80 , dos dijeron que nunca y dos más que cuando se sacaran la lotería; los últīimos dos contestaron que no sabían.

El médico no está consciente de esta situación, entonces el plan para que empiece a tener realmente una aceptación debería ser un plan atractivo, donde se integren todos los médicos y que todos los médicos participen en ese fondo, que tenga un volumen adecuado, que genere confianza, beneficios y seguridad.

Estamos generando un beneficio, este es un proyecto para las generaciones que vienen atrás. Entendemos que no podemos meter al fondo de retiro a todos, pero aproximadamente $40 \%$ de los médicos del hospital ya están en este plan; solamente $15 \%$ son mayores de 60 años, $35 \%$ entre los 40 y 60 años y $100 \%$ de los médicos nuevos que ingresa al hospital.

Otra cosa que se logró con la Asociación Médica es empezar a ver cómo ayudar al aspecto social es decir, la soledad. Se compró una casa en Cuernavaca que tiene ocho habitaciones, la idea es que empēcemos a tener gente que sea mayor y que haya trabajado en el hospital, que pueda vivir ahí y que tēnga un servicio auspiciado por el hospital. 
Lo más importante que logramos a través de estos años es concientizar al médico de este problema real.

\section{Conclusiones}

El plan de jubilación no empieza a construirse cuando el retiro está próximo, sino que es un plan a largo plazo que requiere años de planeación y atención, por ello se empieza cuando se está en plena vida productiva; no consiste en ahorrar lo que nos sobra, es nuestro salario que apartamos para el futuro, intentar apartar para la jubilación. Un importe que nos sobra después de pagar nuestras necesidades y las de nuestra familia es sumamente riesgoso, ya que siempre encontraremos una buena razón para gastar. Este plan es para tener una vida activa en otra etapa de la vida, no es planear para la muerte, es planear para seguir activo, para llevar a cabo actividades que quizás siempre quisimos realizar pero que no tuvimos el tiempo, es calidad de vida.
Por otra parte, la longevidad ha producido la necesidad de que el retiro médico deba considerarse no solo a la luz del juicio de los propios profesionales, sino que deberá ser evaluado por las instituciones en las que trabaja,

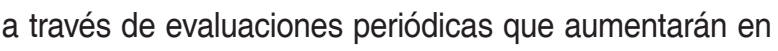
frecuencia al paso del tiempo y que vigilarán las esferas cognitiva, afectiva y psicomotriz, tomando en consideración la especialidad medicaquirúrgica específica.

\section{Lectura recomendada}

Contrato Colectivo de Trabajo 2009-2011. México: Instituto Mexicano dêt Seguro Social/Sindicato Nacional de Trabajadores del Seguro Social;2009.

Fajardo DG. El retiro médico. Introducción. Gac Med Mex. 2011;147:24\&-245.

Gratton L, Scott A. The 100-Year life. Living and working in an age of longevity. Reino Unido: Bloomsbury; 2016.

Martín-Del Campo MN, Sánchez-Marle JF. Plan de retiro médico para conquistar felizmente la vejez. Gac Med Mex. 2011;153:205213.

Muñoz-Kapellman R. El retiro del cirujano. En: Calderón-Abbo M, edítor.Principia quirúrgica. México: Porrúa; 1993.

Muñoz-Kapellmann R. El retiro del médico. Gac Med Mex. 1998;134:327̄336.

Ruiz-Speare JO. El retiro del cirujano: ¿Por qué, cuándo y cómo debe reiirarse un cirujano? An Med Asoc Med Hosp ABC 2018; 63 (1)). 2018;63:73-79.

Sánchez MJ, Ruiz-Contreras EA, Horta BE, Jauregui CL, Martín-Del Campo MN, Wolpert BE, et al. Un análisis del retiro médico en el Centro Mlédico ABC. An Med Asoc Med Hosp ABC. 2013;58:142146.

Wolpert-Barraza E. El retiro clínico-quirúrgico del médico en México. GaĢMed Mex. 2011;147:262-265. 